Vol 11, Issue 10, 2018

\title{
ANTIBIOGRAM PROFILE AND BIOFILM FORMING POTENTIAL OF PSEUDOMONAS SPECIES ISOLATED FROM VARIOUS CLINICAL SPECIMENS
}

\author{
JAMSHEERA CP, ETHEL SUMAN* \\ Department of Microbiology, Kasturba Medical College, Mangaluru, Manipal Academy of Higher Education, Karnataka, India. Email: ethel. \\ suman@manipal.edu
}

Received: 28 February 2018, Revised and Accepted: 11 June 2018

\begin{abstract}
Objective: The present study aimed at finding the resistance pattern of Pseudomonas aeruginosa and other Pseudomonas species isolated from various clinical specimens in the laboratory.

Methods: A total of 150 isolates of different species of Pseudomonas obtained from various clinical specimens processed at the Microbiology laboratory of Kasturba Medical College, Manipal Academy of Higher Education, were taken for this study. Antibiotic susceptibility testing was performed by Kirby-Bauer disc diffusion method and interpreted according to the CLSI guidelines. Biofilm assay was performed by modified O'Toole and Kolter method. The results were analyzed using SPSS 17.0 and Student's unpaired t-test, Kruskal-Wallis, Mann-Whitney, ANOVA, and Chi-square test. $\mathrm{p}<0.05$ was considered statistically significant.
\end{abstract}

Results: Increased resistance was observed by P. aeruginosa to cefotaxime, cotrimoxazole, levofloxacin, ofloxacin, and ticarcillin clavulanate. There was also a good correlation between antibiotic resistance to aztreonam, netilmicin, and ceftazidime and biofilm production. Results of the present study, therefore, demonstrated the occurrence of resistance to various antipseudomonal agents among the biofilm-producing $P$. aeruginosa isolates.

Conclusion: The present study may help in assessing the seriousness of drug resistance caused by biofilm formation in $P$. aeruginosa and devise strategies through antibiotic policies to minimize such problems.

Keywords: Biofilm, Pseudomonas aeruginosa, Antibiogram, Antipseudomonal agents.

(C) 2018 The Authors. Published by Innovare Academic Sciences Pvt Ltd. This is an open access article under the CC BY license (http://creativecommons. org/licenses/by/4. 0/) DOI: http://dx.doi.org/10.22159/ajpcr.2018.v11i10.25518

\section{INTRODUCTION}

Pseudomonas is a large group of aerobic, non-sporing Gram-negative motile rods, which are pervasive in nature. Pseudomonas aeruginosa is a major opportunistic pathogen responsible for acute and chronic infections mainly in hospital settings, especially in patients with compromised host defense mechanism and also with serious underlying disease conditions [1]. According to the CDC, approximately $8 \%$ of all health care-associated infections reported to the National Healthcare Safety Network are caused by P. aeruginosa.

The survival of Pseudomonas within the host in the initial stages of infection is aided by the secretion of various toxins and virulence factors including pyocyanin, proteases, and elastases [2]. P. aeruginosa possesses various intrinsic and acquired mechanisms of drug resistance [3]. Risk factors associated with the emergence of drug-resistant strains include previous antipseudomonal drug treatment and prolonged use of antibiotics. Besides, prolonged hospital stay and increased susceptibility of patients to secondary bacteremia lead to the acquisition of resistant strains [4].

P. aeruginosa forms microcolonies enclosed in extracellular polymeric substances (EPSs) termed as biofilms [5]. Biofilm formation leads to persistent and chronic infection by resisting the action of antimicrobial agents [6]. It has the ability to resist the suppression of the organism by various physical and chemical treatments [5]. Biofilm forms the major response mechanism to external stress factors by inducing many additional phenotypic alterations such as loss of motility, reduced growth rate, and altered susceptibility to host response [6,7].

The present study aimed at correlating biofilm production by $P$. aeruginosa and other species of Pseudomonas isolated from various clinical samples with their antibiogram pattern.

\section{METHODS}

Collection and Identification of bacterial isolates

A total of 150 isolates of Pseudomonas ( $P$. aeruginosa -75 and other Pseudomonas species-75) obtained from various clinical specimens processed at the Microbiology laboratory of Kasturba Medical College, Manipal Academy of Higher Education, were taken for this study (with a $95 \%$ confidence level and $80 \%$ power, the sample size came up to 75 each). The Institutional Ethics Committee clearance was obtained for the study. The isolates were identified by standard biochemical methods [8] or by VITEK 2 system. Antibiotic susceptibility testing was performed by Kirby-Bauer disc diffusion method and interpreted according to the CLSI guidelines [9]. For isolates identified by VITEK 2 system, sensitivity was recorded from it.

\section{Biofilm assay}

This was done by modified 0'Toole and Kolter method [10-13]. The bacterial colony was inoculated in brain heart infusion (BHI) broth and incubated at $37^{\circ} \mathrm{C}$ for $18 \mathrm{~h}$. It was then diluted with fresh BHI broth, and the turbidity was adjusted to $0.5 \mathrm{McF}$ arland standard. $200 \mu \mathrm{l}$ of the suspension was dispensed into microtiter plate wells in duplicate. The plate was incubated at $37^{\circ} \mathrm{C}$ for $24 \mathrm{~h}$. The contents were aspirated and washed with (phosphate-buffered saline( $\mathrm{pH} 7.4)$ following which $100 \mu \mathrm{l}$ bouin fixative was added. The plate was incubated at $25^{\circ} \mathrm{C}$ for $10 \mathrm{~min}$. The contents were discarded, and the wells were stained with $1 \%$ crystal violet. After $1 \mathrm{~min}$, the excess stain was rinsed off by placing the plate under running tap water. Then, $33 \%$ glacial acetic acid was added to each well and optical densities of stained adherent bacterial films were read with Micro ELISA plate reader at $570 \mathrm{~nm}$. Mean reading from two wells were calculated. P. aeruginosa ATCC 27853 was included as control. 
Statistical analysis: All experiments were performed in duplicate. Statistical analysis was performed using SPSS 17.0 for Windows (SPSS, Inc., Chicago, IL, USA) and using Student's unpaired t-test, KruskalWallis, Mann-Whitney, ANOVA, and Chi-square test. $\mathrm{p}<0.05$ was considered statistically significant.

\section{RESULTS}

Of 75 isolates of P. aeruginosa, 64 (35.2\%) were isolated from males, while $11(14.7 \%)$ were isolated from females. With regard to the other Pseudomonas spp., of 75 isolates, 54 (72.0\%) were from males and $21(28.0 \%)$ were from females. As shown in Fig. 1, the highest rate of isolation was from swabs and exudate material from wound infections (21.3\%). Similarly, isolation rate of other species of Pseudomonas also accounted to $24.0 \%$ as shown in Fig. 2 .

\section{Antibiotic resistance of the isolates}

Among the $P$. aeruginosa strains, $80 \%$ were resistant to cefotaxime, $75 \%$ to cotrimoxazole, and $68.2 \%$ to ticarcillin/clavulanic acid. All the isolates were sensitive to colistin and polymyxin-B (Fig. 3). Resistance to levofloxacin was $62.5 \%$. Similarly, the other species of Pseudomonas also showed maximum resistance to cefotaxime (75\%) and cotrimoxazole (75\%), while levofloxacin was third in the list $(66.7 \%)$ as shown in Fig. 4.

\section{Resistance to aminoglycosides}

Among the aminoglycosides, the percentage resistance of $P$. aeruginosa revealed $38.4 \%$ resistance to amikacin, $41.3 \%$ resistance to gentamicin, and also a percentage as high as $46.2 \%$ for netilmicin. However, the percentage of resistance to amikacin was lower in other species of Pseudomonas, being $20.8 \%$ for amikacin, while it was $36.2 \%$ for gentamicin and $12.5 \%$ for netilmicin.

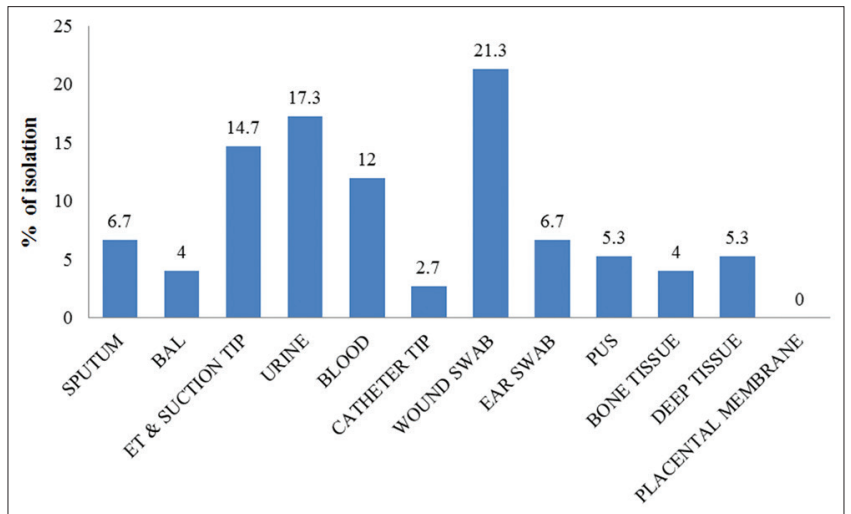

Fig. 1: The percentage isolation of Pseudomonas aeruginosa from various specimens. BAL: Bronchoalveolar lavage

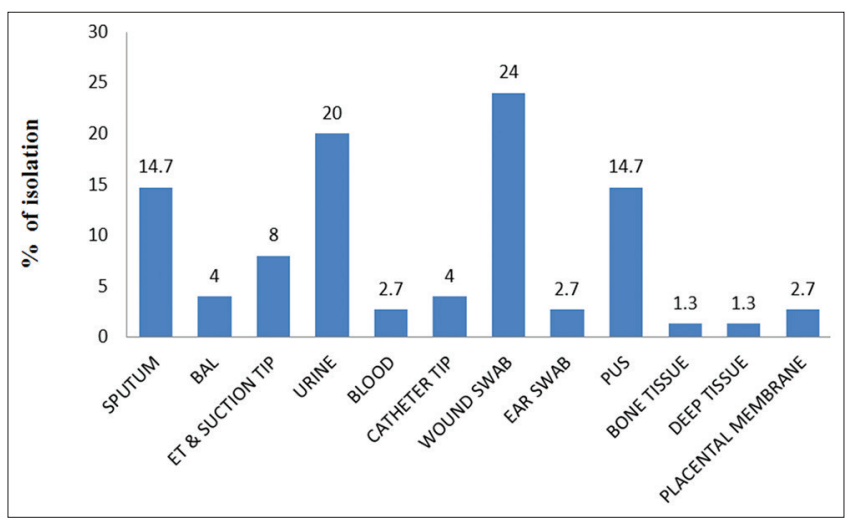

Fig. 2: The percentage isolation of Pseudomonas spp. from various specimens. BAL: Bronchoalveolar lavage

\section{Carbapenem resistance}

P. aeruginosa strains exhibited $24.7 \%$ resistance to imipenem while it was $19.2 \%$ for meropenem which was almost on par with other species of Pseudomonas, and the resistance to imipenem and meropenem being $27 \%$ and $21.1 \%$, respectively.

\section{Resistance to antipseudomonal penicillins}

Most of the strains were sensitive to piperacillin and piperacillintazobactam. Resistance to piperacillin and piperacillin-tazobactam by $P$. aeruginosa was $16.7 \%$ and $12.1 \%$, respectively, while for other species, the percentage of resistance to piperacillin and piperacillintazobactam was $19.6 \%$ and $17.8 \%$, respectively. However, $68.2 \%$ resistance was shown by $P$. aeruginosa against ticarcillin-clavulanic acid, a carboxypenicillin.

\section{Resistance to quinolones}

Both P. aeruginosa and the other species showed high level of resistance to the quinolones with higher resistance to levofloxacin $(62.5 \%$ and $66.7 \%$, respectively), while for ciprofloxacin, the resistance exhibited by P. aeruginosa and the other species was $45.8 \%$ and $34.2 \%$, respectively.

\section{Resistance to minocycline}

There was minimal resistance to minocycline, a tetracycline group of drug by P. aeruginosa strains, while other species showed $100 \%$ susceptibility to this drug.

\section{Resistance to cephalosporins}

The isolates also exhibited resistance to ceftazidime, a third-generation antipseudomonal drug (28.4\% by P. aeruginosa and $23.2 \%$ by the other

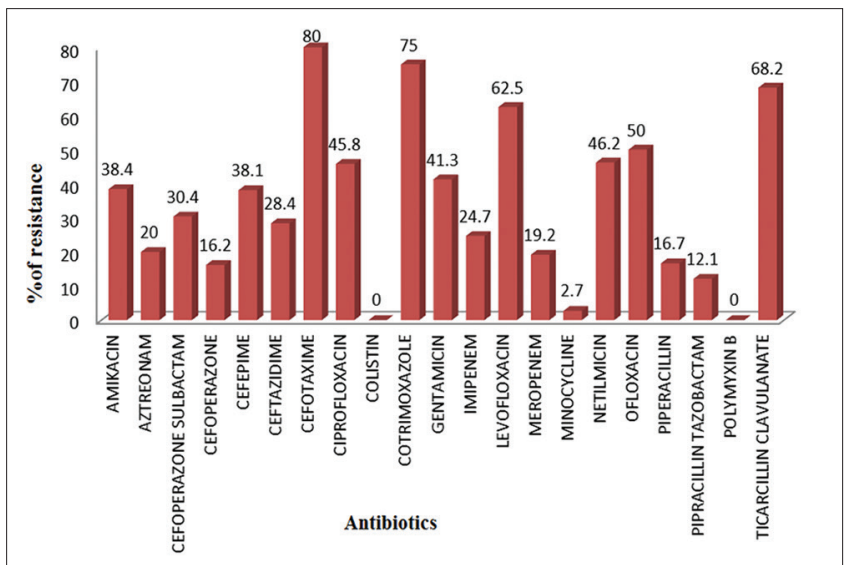

Fig. 3: The resistance pattern of Pseudomonas aeruginosa to various antibiotics

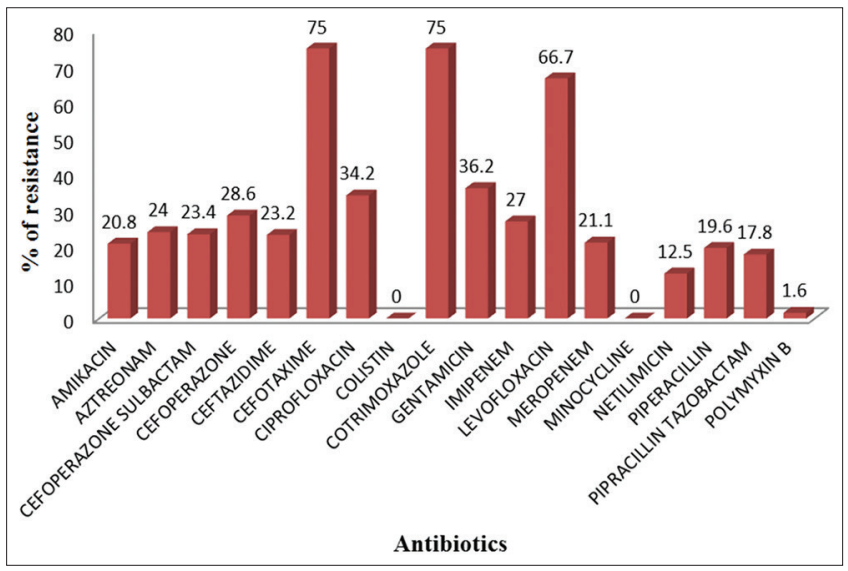

Fig. 4: The resistance pattern of Pseudomonas spp. to various antibiotics 
species). There was a very high percentage of resistance to cefotaxime by $P$. aeruginosa and the other species of Pseudomonas $(80 \%$ and $75 \%$, respectively). The percentage resistance of $P$. aeruginosa and the other species of Pseudomonas to cefoperazone-sulbactam was $30.4 \%$ and $23.4 \%$, respectively, while the resistance to cefoperazone was $16.2 \%$ and $28.6 \%$, respectively.

\section{Resistance to monobactams}

There was $20 \%$ resistance to aztreonam, a monobactam antibiotic by P. aeruginosa, while $24 \%$ resistance was shown by other species of Pseudomonas.

\section{Results of biofilm formation by the isolates}

$\mathrm{OD}_{570}$ of biofilm produced by the isolates is depicted in Tables 1 and 2, and the comparison between the biofilm produced by $P$. aeruginosa and the other species of Pseudomonas is shown in Fig. 5 and Table 3.

\section{Aminoglycosides}

Among the three aminoglycosides, gentamicin- and netilmicin-resistant strains of $P$. aeruginosa showed more biofilm production while amikacin sensitive strains produced more biofilm. In case of other Pseudomonas spp., more biofilm production was shown by aminoglycoside-sensitive strains.

\section{Carbapenems}

P. aeruginosa which was resistant to imipenem produced more amount of biofilm, whereas in other species production, it was more in case of sensitive isolates. With regard to meropenem, intermediately susceptible strains produced more biofilm in case of both P. aeruginosa and Pseudomonas spp.

\section{Antipseudomonal penicillins}

Biofilm production was more by piperacillin susceptible strains of both $P$. aeruginosa and Pseudomonas spp. The strains which were intermediately susceptible to piperacillin-tazobactam isolates showed more biofilm in case of $P$. aeruginosa. In other species, piperacillintazobactam resistant strains produced more biofilm.

\section{Quinolones}

$P$. aeruginosa which was resistant to ciprofloxacin produced more amount of biofilm, whereas in Pseudomonas spp. strains that were intermediately susceptible to ciprofloxacin produced more biofilm. $P$. aeruginosa sensitive to levofloxacin showed more biofilm production, while in case of other species, levofloxacin-resistant ones produced more biofilm.

\section{Cephalosporins}

Isolates of both P. aeruginosa and Pseudomonas spp. which were resistant to cefoperazone sulbactam produced more biofilm. In case of ceftazidime, more biofilm production was showed by P. aeruginosa intermediately susceptible isolates and ceftazidime-resistant isolates of other Pseudomonas spp. Isolates of both P. aeruginosa and Pseudomonas spp. which were resistant to cefotaxime and cefoperazone produced more biofilm.

\section{Monobactams}

P. aeruginosa which was resistant to aztreonam produced more amount of biofilm, whereas in Pseudomonas spp., more biofilm production was in sensitive strains.

Minimum inhibitory concentration (MIC) of certain antibiotics (ceftazidime, ciprofloxacin, and piperacillin-tazobactam) against biofilms also demonstrated that when MIC values increase, there was a significant increase in biofilm.

\section{DISCUSSION}

Antibiotic resistance is a major problem in P. aeruginosa. The organism exhibits intrinsic resistance to several beta-lactam antibiotics and may also acquire additional resistance mechanisms either due to mutational events or due to the acquisition of transferable genetic elements [14].
P. aeruginosa also shows intrinsic resistance by the expression of chromosomally encoded inducible AmpC beta-lactamase and also by several important efflux pump systems that export antibiotics, biocides, dyes, detergents, metabolic inhibitors, organic solvents, and molecules involved in bacterial cell-to-cell communication [15].

Carbapenem resistance mechanisms have emerged under the pressure of carbapenem use in clinical settings and may be classified as enzymatic, which include carbapenemases, aminoglycosidemodifying enzymes, and 16S rRNA methylases or nonenzymatic involving decreased transcription of OprD gene and overproduction of MexAB-OprM efflux system [16]. Carbapenem resistance, however,

Table 1: $\mathrm{OD}_{570}$ of biofilm produced by $P$. aeruginosa isolated from various clinical specimens

\begin{tabular}{lll}
\hline Clinical specimen & Mean number of isolates \pm SD & OD $_{\mathbf{5 7 0}} \mathbf{\pm S D}$ \\
\hline Sputum & $5 \pm 1.023$ & 0.847 \\
Bronchoalveolar lavage & $3 \pm 0.135$ & 0.971 \\
Et and suction tip & $11 \pm 0.473$ & 0.825 \\
Urine & $13 \pm 0.484$ & 0.965 \\
Blood & $9 \pm 0.512$ & 1.069 \\
Catheter tip & $2 \pm 0.520$ & 1.062 \\
Wound swab & $16 \pm 0.439$ & 0.839 \\
Ear swab & $5 \pm 0.554$ & 1.226 \\
Pus & $4 \pm 0.446$ & 0.807 \\
Bone tissue & $3 \pm 0.690$ & 1.434 \\
Deep tissue & $4 \pm 0.323$ & 1.067 \\
Placental membrane & 0 & 0 \\
\hline
\end{tabular}

P. aeruginosa: Pseudomonas aeruginosa

Table 2: $\mathrm{OD}_{570}$ of biofilm produced by Pseudomonas spp. isolated from various clinical specimens

\begin{tabular}{lll}
\hline Clinical specimen & Mean number of isolates $\mathbf{S D D}$ & $\mathbf{O D}_{\mathbf{5 7 0}} \mathbf{\mathbf { S D }}$ \\
\hline Sputum & $11 \pm 0.524$ & 0.989 \\
Bal & $3 \pm 0.393$ & 0.631 \\
Et and suction tip & $6 \pm 0.517$ & 0.925 \\
Urine & $15 \pm 0.387$ & 0.988 \\
Blood & $2 \pm 0.517$ & 1.077 \\
Catheter tip & $3 \pm 0.581$ & 1.202 \\
Wound swab & $18 \pm 0.480$ & 0.866 \\
Ear swab & $2 \pm 0.941$ & 1.339 \\
Pus & $11 \pm 0.499$ & 0.854 \\
Bone tissue & $1 \pm$ & 0.531 \\
Deep tissue & $1 \pm$ & 0.756 \\
Placental membrane & $2 \pm 0.684$ & 2.187 \\
\hline
\end{tabular}

P. aeruginosa: Pseudomonas aeruginosa

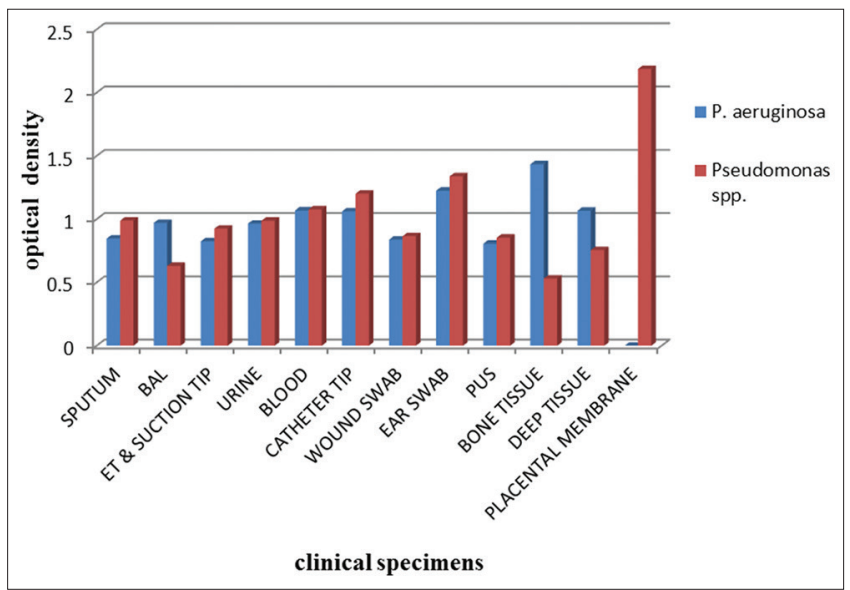

Fig. 5: Comparison of biofilm production in Pseudomonas aeruginosa and Pseudomonas spp. 
Table 3: Correlation between antibiotic sensitivity pattern and biofilm production

\begin{tabular}{|c|c|c|c|c|c|}
\hline Antimicrobial agents & Organism & $\begin{array}{l}\text { Mean OD } \\
\text { Sensitive }\end{array}$ & $\begin{array}{l}\text { Mean } 0 D_{570} \text { isolates } \pm S D \\
\text { Resistant }\end{array}$ & $\begin{array}{l}\text { Mean } O D_{570} \text { isolates } \pm S D \\
\text { Intermediate }\end{array}$ & p value \\
\hline \multirow[t]{2}{*}{ Amikacin } & P. aeruginosa & $0.975 \pm 0.471$ & $0.966 \pm 0.58$ & & 0.941 \\
\hline & Pseudomonas spp. & $1.03 \pm 0.553$ & $0.758 \pm 0.347$ & $0.807 \pm 0.089$ & 0.166 \\
\hline \multirow[t]{2}{*}{ Ceftazidime } & P. aeruginosa & $0.905 \pm 0.458$ & $0.923 \pm 0.551$ & $1.491 \pm 0.492$ & 0.015 \\
\hline & Pseudomonas spp. & $0.970 \pm 0.507$ & $1.067 \pm 0.600$ & $0.694 \pm 1$ & 0.698 \\
\hline \multirow[t]{2}{*}{ Cefoperazone sulbactam } & P. aeruginosa & $0.941 \pm 0.466$ & $1.131 \pm 0.614$ & $0.708 \pm 0.410$ & 0.242 \\
\hline & Pseudomonas spp. & $0.983 \pm 0.492$ & $1.005 \pm 0.592$ & $0.871 \pm 0.603$ & 0.901 \\
\hline Ciprofloxacin & Pseudomonas spp. & $0.968 \pm 0.490$ & $0.932 \pm 0.539$ & $2.08 \pm 1$ & 0.093 \\
\hline \multirow[t]{2}{*}{ Gentamicin } & P. aeruginosa & $0.888 \pm 0.454$ & $1.059 \pm 0.588$ & $0.873 \pm 0.191$ & 0.367 \\
\hline & Pseudomonas spp. & $1.046 \pm 0.546$ & $0.852 \pm 0.466$ & $0.951 \pm 1$ & 0.337 \\
\hline \multirow[t]{2}{*}{ Imipenem } & P. aeruginosa & $0.886 \pm 0.433$ & $1.194 \pm 0.682$ & & 0.027 \\
\hline & Pseudomonas spp. & $1.014 \pm 0.573$ & $0.832 \pm 0.347$ & $1.014 \pm 0.099$ & 0.408 \\
\hline \multirow[t]{2}{*}{ Meropenem } & P. aeruginosa & $0.959 \pm 0.508$ & $0.864 \pm 0.489$ & $1.442 \pm 1$ & 0.507 \\
\hline & Pseudomonas spp. & $1.004 \pm 0.550$ & $0.783 \pm 0.331$ & $1.396 \pm 0.968$ & 0.183 \\
\hline Piperacillin & P. aeruginosa & $0.891 \pm 0.454$ & $0.972 \pm 0.432$ & & 0.647 \\
\hline \multirow[t]{2}{*}{ Piperacillin-tazobactam } & P. aeruginosa & $0.961 \pm 0.524$ & $0.784 \pm 0.398$ & $1.466 \pm 1$ & 0.395 \\
\hline & Pseudomonas spp. & $0.962 \pm 0.482$ & $1.044 \pm 0.677$ & $0.335 \pm 1$ & 0.419 \\
\hline \multirow[t]{2}{*}{ Netilmicin } & P. aeruginosa & $0.805 \pm 0.190$ & $1.289 \pm 0.529$ & & 0.044 \\
\hline & Pseudomonas spp. & $1.080 \pm 0.491$ & $0.717 \pm 0.094$ & & 0.328 \\
\hline \multirow{2}{*}{ Cotrimoxazole } & P. aeruginosa & $0.709 \pm 0.050$ & $1.175 \pm 0.951$ & & - \\
\hline & Pseudomonas spp. & $1.340 \pm 1$ & $0.878 \pm 0.161$ & & - \\
\hline \multirow[t]{2}{*}{ Aztreonam } & P. aeruginosa & $0.776 \pm 0.337$ & $1.613 \pm 0.783$ & & 0.003 \\
\hline & Pseudomonas spp. & $1.007 \pm 0.582$ & $0.874 \pm 0.437$ & & 0.614 \\
\hline \multirow[t]{2}{*}{ Cefotaxime } & P. aeruginosa & & $1.11 \pm 0.674$ & $0.744 \pm 1$ & - \\
\hline & Pseudomonas spp. & $0.738 \pm 1$ & & $0.934 \pm 0.441$ & - \\
\hline \multirow[t]{2}{*}{ Cefoperazone } & P. aeruginosa & $0.891 \pm 0.443$ & $1.097 \pm 0.681$ & $0.392 \pm 0.081$ & 0.211 \\
\hline & Pseudomonas spp. & $0.907 \pm 0.460$ & $1.059 \pm 0.681$ & $0.896 \pm 1$ & 0.709 \\
\hline \multirow[t]{2}{*}{ Levofloxacin } & P. aeruginosa & $1.473 \pm 0.508$ & $1.251 \pm 0.688$ & $0.771 \pm 0.084$ & 0.451 \\
\hline & Pseudomonas spp. & $0.531 \pm 1$ & $0.845 \pm 0.213$ & & 0.996 \\
\hline
\end{tabular}

P. aeruginosa: Pseudomonas aeruginosa

develops frequently due to the concomitant presence of more than one mechanism $[17,18]$.

The present study showed a higher level of imipenem resistance as compared to meropenem resistance. This could also be due to the frequent use of imipenem which could change the permeability of the outer membrane or modify the target sites of the organism when given with piperacillin, thus resulting in carbapenem resistance [19]

Most of the isolates were sensitive to aztreonam, the only monobactam possibly effective against carbapenem-hydrolyzing strains of $P$. aeruginosa which is often used in patients who are penicillin allergic or who cannot tolerate aminoglycosides [20].

Previous studies have shown growing resistance to ciprofloxacin and cefotaxime by $P$. aeruginosa [21-24]. This was quite similar to the resistance pattern that was observed in our study with resistance percentage to cefotaxime being as high as $80 \%$, while for ciprofloxacin, it was $45.8 \%$. Ceftazidime was shown a high level of resistance in previous study [1], but the present study shows a decrease in resistance pattern

Quinolones are generally preferred by the physicians as an empirical therapy mainly because of the easy availability in oral forms and reasonable cost [25-27].

However, emerging quinolone-resistant strains of $P$. aeruginosa have surfaced owing to the changes in target enzymes of the bacteria and active efflux pumps generated to prevent the entry of the drugs. The present study also found very high resistance to levofloxacin and ciprofloxacin by $P$. aeruginosa

The present study also showed a high level of resistance to ticarcillinclavulanic acid, a carboxypenicillin. Studies have demonstrated that clavulanate may induce AmpC expression in P. aeruginosa [28]. Significant induction was shown to occur with pharmacokinetically relevant concentrations of clavulanate. Besides, the induction of $\mathrm{AmpC}$ by clavulanate was shown to significantly antagonize or substantially diminish the antibacterial activity of ticarcillin. The study also suggested that, in the selection of an antipseudomonal $\beta$-lactam for the treatment of $P$. aeruginosa infections, the combination of ticarcillin-clavulanate should be avoided, especially with immunocompromised patients, for whom bacterial killing is required to ensure clinical success.

It was interesting to note the correlation between antibiotic resistance and biofilm production. Among the aminoglycosides, netilmicin resistance and biofilm production demonstrated good correlation $(p=0.044)$. Aminoglycosides which constitute a vital component of antipseudomonal therapy have been showing resistance in the recent past. Hence, it is important to remember that prolonged therapy may lead to persisters by forming biofilms [29].

Imipenem-resistant $P$. aeruginosa strains produced more amount of biofilm. Imipenem exposure may lead to an expression of alginate which is known to provide the biofilm bacteria with a protected environment [30].

Aztreonam, the monobactam commonly preferred for treating Pseudomonas infections in patients allergic to penicillins or aminoglycosides, showed only $20 \%$ resistance, but the resistant strains were strong biofilm producers $(\mathrm{p}=0.003)$. It is possible that resistance may lead to persister cells in this case and this needs to be studied further.

Inhaled aztreonam has been used in combination with tigecycline in treatment of cystic fibrosis, and the study has also shown enhancement of antibiofilm activity. However, some strains have shown tolerance to aztreonam-tigecycline combination [31]. Ps1EPS production appears to relate to antibiotic tolerance in some of the strains. In addition to this, other mechanisms such as lower bacterial outer membrane permeability, antibiotic efflux pump, and beta-lactamase resistance may play a role in $P$. aeruginosa tolerance to aztreonam [32,33]. In a particular study, even same antibiotics showed significant quorum sensing inhibitory activity 
against different test organisms, and in clinical isolates, similar antibiotics exhibited varied effects in their effective concentration[34,35].

Our study revealed that isolates of both P. aeruginosa and Pseudomonas spp. which were resistant to cefotaxime and cefoperazone produced more biofilm. A particular study has shown the ability of cefuroxime to dislodge the biofilm formation in Pseudomonas by $65.57 \%$.

Increase in biofilm with an increase in MIC of antibiotics seen in the present study is on par with other studies reported $[10,13,14]$

\section{CONCLUSION}

Results of the present study demonstrated the occurrence of resistance to various antipseudomonal agents among the P. aeruginosa isolates as well as in other species of Pseudomonas. While certain drugs such as piperacillin-tazobactam, carbapenems, and amikacin remain the mainstay of the treatment of pseudomonal infections, it is alarming to note an increase in resistance levels to these antibiotics. Besides this, the organisms also produce biofilms which serve as barriers to effective therapy. Regular antimicrobial susceptibility monitoring would help and guide the physicians in prescribing the right combinations of antimicrobial to limit and prevent the emergence of multidrugresistant strains of $P$. aeruginosa. Antibiotics should be used judiciously and at the optimum concentration so as to inhibit biofilm formation and eradicate persister cells. Studies have postulated that combination of a biofilm inhibitor with a conventional antibiotic to control biofilms thereby permits the drug to reach the cells trapped inside the biofilm. As this is a hospital-based epidemiological data, the present study will help in implementation of better patient management and infection control strategies.

\section{CONFLICTS OF INTEREST}

All authors have none to declare.

\section{REFERENCES}

1. Anvarinejad M, Japoni A, Rafaatpour N, Mardaneh J, Abbasi P, Shahidi MA, et al. Burn patients wounds infected with metallo-betalactamase-producing Pseudomonas aeruginosa: Multidrug resistant strains. Arch Trauma Res 2014;3:e18182.

2. Van 't Wout E, van Schadewijk A, van Boxtel R, Dalton L, Clarke H, Tommassen $\mathrm{J}$, et al. Virulence factors of Pseudomonas aeruginosa induce both the unfolded protein and integrated stress responses in airway epithelial cells. PLoS Pathog 2015;11:e1004946.

3. Mendiratta D, Narang P, Buchunde S, Deotale V. Comparison of disc and MIC reduction methods with polymerase chain reaction for the detection of metallo- $\beta$-lactamase in Pseudomonas aeruginosa. Indian J Med Microbiol 2012;30:170

4. Peña C, Gómez-Zorrilla S, Suarez C, Dominguez MA, Tubau F, Arch O, et al. Extensively drug-resistant Pseudomonas aeruginosa: Risk of bloodstream infection in hospitalized patients. Eur J Clin Microbiol Infect Dis 2012;31:2791-7.

5. Lambert PA. Mechanisms of antibiotic resistance in Pseudomonas aeruginosa. J R Soc Med 2002;95:22-6.

6. Wei Q, Ma LZ. Biofilm matrix and its regulation in Pseudomonas aeruginosa. Int J Mol Sci 2013;14:20983-1005.

7. Kaiser S, Mutters N, De Rosa A, Ewers C, Frank U, Günther F. Determinants for persistence of Pseudomonas aeruginosa in hospitals: Interplay between resistance, virulence and biofilm formation. Eur J Clin Microbiol Infect Dis 2017;36:243-53.

8. Collee J, Mackie T, McCartney J. Mackie and McCartney Practical Medical New York : Churchill Livingstone; 1996. p. 151-8.

9. CLSI. Performance Standards for Antimicrobial Susceptibility Testing. Informational Supplement CLSI Document. M100-S26. $26^{\text {th }}$ ed. Wayne, PA: Clinical and Laboratory Standards Institute; 2008.

10. O'Toole GA, Kolter R. Initiation of biofilm formation in Pseudomonas fluorescens WCS365 proceeds via multiple, convergent signalling pathways: A genetic analysis. Mol Microbiol 1998;28:449-61.

11. Suman E, Varghese B, Joseph N, Nisha K, Kotian MS. The Bacterial biofilms in dialysis water systems and the effect of sub-inhibitory concentrations of chlorine on them. J Clin Diagn Res 2013;7:849-52.

12. Suman E, Jose J, Varghese S, Kotian MS. Study of biofilms production in Escherichia coli causing urinary tract infection. Ind J Med Microbiol 2007;25:305-6.

13. Suman E, Singh S, Kotian MS. Pseudomonas aeruginosa biofilms in hospital water systems and the effect of sub-inhibitory concentration of chlorine. J Hosp Infect 2008;70:199-201.

14. Meletis G, Exindari M, Vavatsi N, Sofianou D, Diza E. Mechanisms responsible for the emergence of carbapenem resistance in Pseudomonas aeruginosa. Hippokratia 2012;16:303-7.

15. Livermore DM. Multiple mechanisms of antimicrobial resistance in Pseudomonas aeruginosa: Our worst nightmare? Clin Infect Dis 2002;34:634-40.

16. Poole K. Pseudomonas aeruginosa: Resistance to the max. Front Microbiol 2011;2:65

17. Hammami S, Ghozzi R, Burghoffer B, Arlet G, Redjeb S. Mechanisms of carbapenem resistance in non-metallo-beta-lactamase-producing clinical isolates of Pseudomonas aeruginosa from a Tunisian hospital. Pathol Biol (Paris) 2009;57:530-5.

18. El Amin N, Giske CG, Jalal S, Keijser B, Kronvall G, Wretlind B. Carbapenem resistance mechanisms in Pseudomonas aeruginosa: Alterations of porin OprD and efflux proteins do not fully explain resistance patterns observed in clinical isolates. APMIS 2005;113:187-96.

19. Strateva T, Yordanov D. Pseudomonas aeruginosa - A phenomenon of bacterial resistance. J Med Microbiol 2009;58:1133-48.

20. Walton MA, Villarreal C, Herndon DN, Heggers JP. The use of aztreonam as an alternate therapy for multi-resistant Pseudomonas aeruginosa. Burns 1997;23:225-7.

21. Khatri B, Basnyat S, Karki A, Poudel A, Shrestha B. Etiology and antimicrobial susceptibility pattern of bacterial pathogens from urinary tract infection. Nepal Med Coll J 2012;14:129-32.

22. Zakaria EA. Increasing ciprofloxacin resistance among prevalent urinary tract bacterial isolates in Gaza Strip, Palestine. J Biomed Biotechnol 2005;3:238-41.

23. Naeem M, Khan MA, Qazi SM. Antibiotic susceptibility pattern of bacterial pathogens causing urinary tract infection in a tertiary care hospital. Ann Pak Inst Med Sci 2010;6:214-8.

24. Hasan AS, Nair D, Kaur J, Baweja G, Deb M, Aggarwal P. Resistance patterns of urinary isolate in a tertiary Indian hospital. J Ayub Med Coll Abbottabad 2007;19:39-41

25. Chikwendu CI, Amadi ES, Obi RK. Prevalence and antimicrobial resistance in Pseudomonas aeruginosa and Klebsiella pneumoniae isolates from non-clinical urine samples. New York Sci J.2010;3:194-200.

26. Drago L, De Vecchi E, Mombelli B, Nicola L, Valli M, Gismondo MR. Activity of levofloxacin and ciprofloxacin against urinary pathogens. J Antimicrob Chemother 2001;48:37-45.

27. Prakash D, Saxena RS. Distribution and antimicrobial susceptibility pattern of bacterial pathogens causing urinary tract infection in urban community of Meerut City, India. ISRN Microbiol 2013;2013:749629.

28. Lister P D, Gardner V M, Sanders CC. Clavulanate induces expression of the Pseudomonas aeruginosa AmpC cephalosporinase at physiologically relevant concentrations and antagonizes the antibacterial activity of ticarcillin. Antimicrob Agents Chemother 1999;4:882-9.

29. Poole K. Aminoglycoside resistance in Pseudomonas aeruginosa antimicrob. Agents Chemother 2005;49:479-87.

30. Bagge N, Schister M, Hentzer M, Ciofu O, Givskov M, Greenberg EP, et al. Pseudomonas aeruginosa biofilms exposed to imipenem exhibit changes in global gene expression and $\beta$-Lactamase and alginate production. Antimicrob Agents Chemother 2004;48:1175-87.

31. Rojo-Molinero E, Macia M, Rubio R, Moya B, Cabot G, Lopez-Causape C. Sequential treatment for biofilms with aztreonam and tobramycin is a novel strategy for combating Pseudomonas aeruginosa chronic respiratory infections. Antimicrobial Agents Chemother 2016;60:2912-22

32. Hancock RE. Resistance mechanisms in Pseudomonas aeruginosa. Clin Infect Dis 1998;27 Suppl 1:S93-9.

33. Yu Q, Griffin EF, Moreau-Marquis S, Shwartzman JD, Stanton BA, O'Toole GA. In vitro evaluation of tobramycin and aztreonam versus Pseudomonas aeruginosa biofilms oncystic fibrosis derived human airway epithelial cells. J Antimicrob Chemother 2012;67:2673-81.

34. Mary RN, Banu N. Screening of antibiofilm and anti quorum sensing potential of Vitex trifolia in Pseudomonas aeruginosa. Int J Pharm Sci 2015;7:242-5

35. Rathinam P, Viswanathan P. Effects of antibiotics upon quorum sensing regulated characters: A propitious scheme against device associated infections. Int J Pharm Pharm Sci 2014;6:85-90. 